\title{
Trigeminal neuralgia: successful antiepileptic drug combination therapy in three refractory cases
}

This article was published in the following Dove Press journal:

Drug, Healthcare and Patient Safety

9 August 20II

Number of times this article has been viewed

\author{
Lara Prisco' \\ Mario Ganau ${ }^{2}$ \\ Federica Bigotto' \\ Francesca Zornada' \\ 'Department of Anaesthesiology, \\ Intensive Care and Emergency \\ Medicine, University Hospital \\ of Cattinara, ${ }^{2}$ Graduate School \\ of Nanotechnology, University \\ of Trieste, Italy
}

\begin{abstract}
Antiepileptic drug combination therapy remains an empirical second-line treatment approach in trigeminal neuralgia, after treatment with one antiepileptic drug or other nonantiepileptic drugs have failed. The results in three patients followed in our clinic are not sufficient to draw definitive conclusions, but suggest the possibility of developing this type of therapeutic approach further.
\end{abstract}

Keywords: trigeminal neuralgia, antiepileptic drugs, combination therapy

\section{Introduction}

The annual incidence of trigeminal neuralgia is approximately 12.6 new cases per 100,000 people per year, with a female to male preponderance of $3: 2{ }^{1}$ Trigeminal neuralgia is defined by the International Classification of Headache Disorders as paroxysmal attacks of pain (strong, sharp, superficial, or stabbing) lasting from a fraction of a second to 2 minutes, precipitated by stimulation of "trigger zones" or triggers with involvement of one or more divisions of the trigeminal nerve. The age of onset is 40-60 years for classic trigeminal neuralgia (idiopathic, not attributed to another disorder) and 30-40 years for symptomatic trigeminal neuralgia (secondary to compression of the trigeminal ganglion or to a demyelinating disorder).

Several studies have investigated carbamazepine, gabapentin, and pregabalin for their effectiveness in the treatment of trigeminal neuralgia. ${ }^{2-4}$ Currently, the combination of antiepileptic drugs in the treatment of trigeminal neuralgia is a "second-line approach." Antiepileptic drug treatment is sometimes considered when a combination of nonantiepileptic drugs fails. We report here three patients with trigeminal neuralgia who were successfully treated using a combination of antiepileptic drugs after failure of first-line and other therapeutic strategies.

\section{Case series}

The records of three patients $(\mathrm{A}, \mathrm{B}$, and $\mathrm{C}$ ) diagnosed with trigeminal neuralgia and attending the Clinic for Pain Therapy at our institution were retrospectively reviewed, and appropriate information was collected. These patients were chosen as cases to report because of their refractory typical trigeminal neuralgia symptoms. Patients who did not require a combination of antiepileptic drugs to treat their symptoms were not included. The patients provided written consent for their information to be used for clinical research. Two patients were found to have trigeminal neuralgia secondary to ganglion compression (A and B) and the third patient had an idiopathic form $(\mathrm{C})$.
Correspondence: Lara Prisco

Via San Francesco 12 ,

Trieste 34133, Italy

Tel +393402293558

$\mathrm{Fax}+39040823375$

Email priscolara@gmail.com
Drug, Healthcare and Patient Safety 201 I:3 43-45

(C) 20II Prisco et al, publisher and licensee Dove Medical Press Ltd. This is an Open Access article

Dovepress

http://dx.doi.org//0.21 147/DHPS.S22385 
The pathophysiological characteristics and therapeutic management of the patients are summarized in Table 1. The two patients with secondary trigeminal neuralgia underwent neurosurgical decompression of the trigeminal ganglion and other minimally invasive treatment (nerve block and infiltration), in addition to several sessions of acupuncture which did not change either in intensity or type of pain. In a different tertiary clinic, all three patients had in the first instance taken antiepileptic monotherapy using various combinations of nonantiepileptic drugs for a period of 7 months to 8 years without obtaining satisfactory results in terms of pain relief, but experiencing various side effects due to the use of antidepressants (drowsiness), opioids (nausea and constipation), and nonsteroidal anti-inflammatory drugs (epigastralgia).

The three patients were subsequently prescribed a combination of antiepileptic drugs for a period of 1-7 months. Carbamazepine was prescribed with gabapentin or pregabalin, taking advantage of the different actions of these agents at various receptor levels. The dosages of all the drugs were lower (see Table 1) than the therapeutic dose of each drug when used alone, without the need for initial titration, thereby decreasing the potential risk of side effects. All patients underwent regular blood investigations to ensure consistent carbamazepine levels, which were always within the therapeutic range (4-12 mg/L).

All patients showed a marked clinical improvement on combination therapy (carbamazepine + gabapentin or carbamazepine + pregabalin) and decided voluntarily to reduce or suspend treatment after remission of pain symptoms, and have not reported recurrence of symptoms since their last follow-up visits in our clinic at 1 year. There have been no side effects reported following the combination therapy. Only one patient complained of dizziness, which predated the start of the antiepileptic drug combination.

\section{Discussion}

Trigeminal neuralgia causes episodes of paroxysmal pain that are short-lasting but intense in nature. The intervals between the paroxysms are generally free from painful symptoms, but a constant dull pain persists in some cases. ${ }^{5}$ Medical treatment of trigeminal neuralgia includes the use of antiepileptic drugs (carbamazepine, gabapentin, pregabalin, lamotrigine) and nonantiepileptic drugs (baclofen, tocainide, pimozide, clomipramine, amitriptyline, tizanidine, proparacaine). The most studied antiepileptic drugs in trigeminal neuralgia are carbamazepine, baclofen, lamotrigine, and pimozide. ${ }^{6}$ Recent studies have suggested the use of other antiepileptic drugs in trigeminal neuralgia, such as

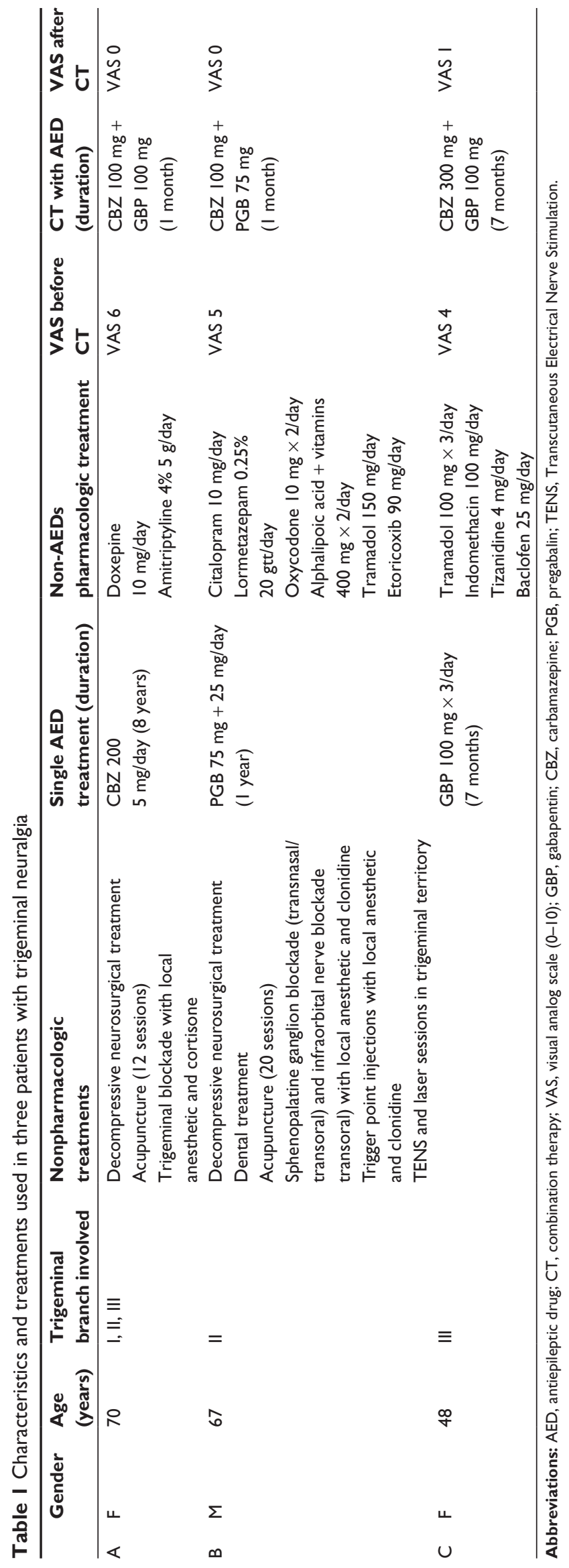


gabapentin and pregabalin., ${ }^{2,7}$ No study has ever compared antiepileptic drug monotherapy with combination therapy of two antiepileptic drugs, exploiting their different actions at the synaptic level, although in one study polypharmacy has been used successfully as second-line treatment after eight weeks of unsuccessful monotherapy. ${ }^{2}$ It is assumed that carbamazepine controls paroxysmal pain by suppression of ectopic neuronal transmission, ie, blocking synaptic sodium channels, whereas gabapentin and pregabalin interact with the $\alpha_{2} \delta$ subunit of voltage-gated calcium channels by increasing the brain concentration and rate of synthesis of gamma aminobutyric acid..$^{8,9}$

There are no randomized trials demonstrating the effectiveness of a combination of anticonvulsants compared with monotherapy or following unsuccessful monotherapy in their study of pregabalin. In their study of pregabalin, Obermann et $\mathrm{al}^{2}$ added carbamazepine $600-1200 \mathrm{mg}$ /day or lamotrigine $50-200 \mathrm{mg} /$ day after eight weeks of pregabalin monotherapy. Although their research did not investigate the efficacy of combination therapy, the results showed a marked reduction of pain in two patients treated with anticonvulsants. The three patients followed in our clinic are not sufficient to draw any kind of conclusion, but suggest the possibility of further investigation of this type of therapeutic approach.

\section{Disclosure}

The authors report no conflicts of interest in this work.

\section{References}

1. Koopman J, de Vries L, Dieleman J, et al. A nationwide study of three invasive treatments for trigeminal neuralgia. Pain. 2011;152:507-513.

2. Obermann M, Yoon MS, Sensen K, et al. Efficacy of pregabalin in the treatment of trigeminal neuralgia. Cephalalgia. 2008;28:174-181.

3. Taylor JC, Brauer S, Espir ML, et al. Long term treatment of trigeminal neuralgia with carbamazepine. Postgrad Med J. 1981;57:16-18.

4. Magnus L. Nonepileptic uses of gabapentin. Epilepsia. 1999;40(S): S66-S72.

5. Headache Classification Committee of the International Headache Society. The International Classification of Headache Disorders. 2nd ed. Cephalalgia. 2004;24:1-160.

6. Gronseth G, Cruccu G, Alksne J, et al. Practice parameter: The diagnostic evaluation and treatment of trigeminal neuralgia (an evidence-based review): Report of the Quality Standards Subcommittee of the American Academy of Neurology and the European Federation of Neurological Societies. Neurology. 2008;71:1183-1190.

7. Rozen TD. Antiepileptic drugs in the management of cluster headache and trigeminal neuralgia. Headache. 2001;41:S25-S32.

8. Guay DR. Pregabalin in neuropathic pain: A more 'pharmaceutically elegant' gabapentin? Am J Geriatr Pharmacother. 2005;3:274-287.

9. Taylor CP, Gee NS, Su TZ, et al. A summary of mechanistic hypotheses of gabapentin pharmacology. Epilepsy Res. 1998;29:233-249.
Drug, Healthcare and Patient Safety

\section{Publish your work in this journal}

Drug, Healthcare and Patient Safety is an international, peer-reviewed open-access journal exploring patient safety issues in the healthcare continuum from diagnostic and screening interventions through to treatment, drug therapy and surgery. The journal is characterized by the rapid reporting of reviews, original research, clinical, epidemiological and

\section{Dovepress}

post-marketing surveillance studies, risk management, health literacy and educational programs across all areas of healthcare delivery. The manuscript management system is completely online and includes a very quick and fair peer-review system. Visit http://www.dovepress.com/ testimonials.php to read real quotes from published authors. 